\title{
Molecular Modeling and Biochemical Studies of Transglutaminase 2 Mutation Found in Patients with Early-onset Type 2 Diabetes
}

\author{
Jung-Mo Kim, ${ }^{\circledR}$ Younho Lee, ${ }^{\dagger, \downarrow}$ Nam Doo Kim, Jeong Hyeok Yoon, ${ }^{\ddagger}$ Yoongho Lim, ${ }^{\ddagger}$ \\ Kyung-Chae Jeong, Chang-Hoon Lee, Soo-Youl Kim, and Byung Il Lee* \\ Molectlar Oncologv Branch, Division of Basic and Applied Sciences, Research Institute, Kational Cancer Center, \\ Govang, Gyeonggi 10-769, Korea. E-mail: bilee Gincc.re.kr \\ ${ }^{\dagger}$ Equispharm Co, Ltd, 86t-1, Iui-dong. Ieongtong-gu, Stw'on, Gieonggi 4+3-766, Korea \\ ${ }^{\ddagger}$ Division of Bioscience and Biotechnologv, BMIIC, Research Center for Drugs, Konkuk Uniwersitw, Seoul 143-701, Korea
} Received June 30, 2008

Key Words : Transglutaminase 2. I- $\kappa \mathrm{B} \alpha$. Diabetes. Modeling

Transglutaminase 2 is a calcium-dependent multi-functional protein that catalyzes the formation of $\mathrm{N} \varepsilon-(\gamma$-glutamyl)lysine isopeptide bond between lysine and glutanine residues. ${ }^{1}$ Transglutaminase 2 induces Nuclear Factor $-\kappa B$ (NF- $\kappa$ B) activation through the polymerization of I- $\kappa \mathrm{B} \alpha$ which results in anti-apoptotic cellular function ${ }^{2, \hat{3}}$ It is highly associated with inflanmmatory diseases and various cancers. ${ }^{+,-}$

Recently. three types of missense mutations (M330R. $1331 \mathrm{~N}, \mathrm{~N} 333 \mathrm{~S}$ ) in transglutaminase 2 were found in patients with early-onset type 2 diabetes. Mutated residues, moreover, were located near the catalytic site, and the nutations resulted in loss of the transamidation activity of transglutaminase 2 from the in vitro analysis. ${ }^{6}$ To explain why these nutants do not have transanidation activity in vitor. we analyzed the structural basis of functional loss in mutants using the molecular modeling method. The in wivo analysis of functional loss of mutants was studied by monitoring the substrate $\mathrm{I}-\kappa \mathrm{B} \alpha$ level in cytosolic fraction of PANC-I cells.

Structural basis of mutation and loss of function by molecular modeling. Poirzio et al. reported that three mutations in early-onset type 2 diabetes lacked their transanidation activity. ${ }^{6}$ The structural basis of the loss of function was analyzed by the molecular modeling method. A five-amino-acid sequence around the glutamine 182 $\left(\mathrm{SQ}^{18} \mathrm{SKV}\right)$ of $\beta$-casein was used as substrate for modeling. ${ }^{7}$

The crystal structure of the active transglutaminase 2 complex with the peptide inhibitor has been reported ${ }^{s}$ The docking models were determined using the inhibitor (Ac$\left.\mathrm{P}(\mathrm{DON}) \mathrm{LPF}-\mathrm{NH}_{2}\right)$ and $\mathrm{X}$-ray crystal structure of transglutaminase $2(2 \mathrm{Q} 3 \mathrm{Z})$ as a template (Figure 1A).

We also derived several transglutaminase $2: \mathrm{I}-\mathrm{B} \mathrm{B} \alpha \mathrm{com}$ plex models using the $\mathrm{IQ}^{166} \mathrm{QQL}$. $\mathrm{QQ}{ }^{267} \mathrm{QLG}$ and $\mathrm{GQ}^{31{ }^{3}} \mathrm{RLT}$ sequences, respectively.

The length between the M330 of the transglutaminase 2 and the proline of the inhibitor (Ac-P(DON)LPF-NH2) is about $4.3 \AA$ as shown in the open form of the transglutaninase 2 crystal structure (2Q3Z: Figure 1A). The M330 residue forms a proline interaction pocket and inter-

IM Kim and Y Lee contributed equally to this work: acts with the inhibitor. In our model using $\beta$-casein, the $\mathrm{Q}+2$ position residue of the substrate can interact with the M330 residue, and a change of the M330 residue by another polar amino acid can affect the function of transglutaminase 2 (Figure IB).

I331 residue is one of the residues at the hydrophobic pocket. which is also related to inhibitor binding. The phenyl residue of the inhibitor (Ac-P(DON)LPF-NH2) binds to the hydrophobic pocket formed by the A304, L312. I313. F316, I331 and $L 420$ residues. ${ }^{\circ}$ Transglutaminase 2 preferred the hydrophobic residue at the $\mathrm{Q}+3$ position from the glutamine residue of substrates. ${ }^{10}$ Thus. this pocket is strongly related

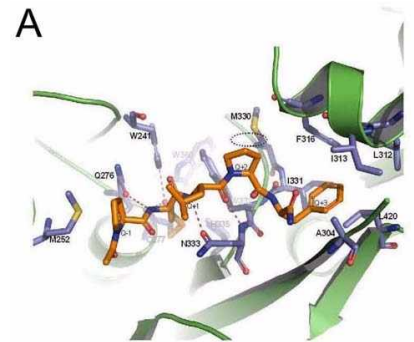

2Q3Z crystal structure C

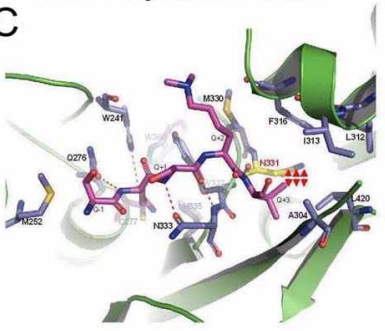

TG2 I331N:Casein

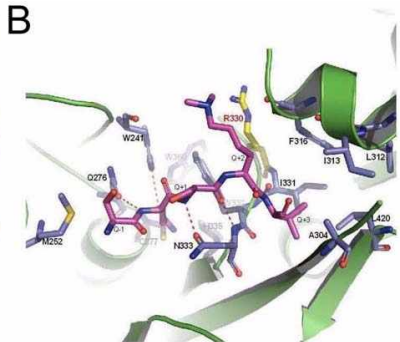

TG2 M330R:Casein

$\mathrm{D}$

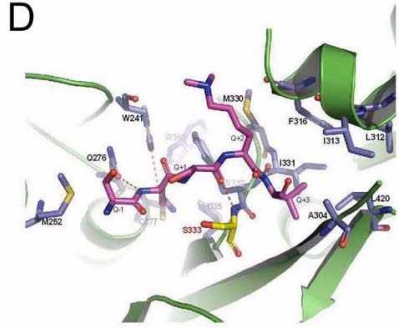

TG2 N333S:Casein
Figure 1. Crystal structure complex with inhibitor and substrate binding model for three types of mutants. (A) Active sites of transglutaminase 2 (TG2) complex with inhibitor (Ac-P(DON)LPF$\mathrm{NH} 2{ }^{8}{ }^{8} \mathrm{M} 330$ 's interaction with the proline residue of the inhibitor is shown in the circle. (B) M330R mutant model of transglutalminase 2 with $A, N$-dimethyl $\beta$-casein. (C) $\beta$-asein binding model in $I 33 \mathrm{IN}$ mutant showing that valine residue of $\beta$ casein did not interact with N331. (D) N333S mutant loses hydrogen-bonding with backbone ' $\mathrm{NH}$ ' of substrate. The peptide inhibitor of the crystal structure is represented by the orange stick, and the $\mathrm{SQ}^{182} \mathrm{SKV}$ sequence of $\beta$ casein, by the magenta stick. 
to the specificity for the substrate. In the I331N mutation. the interaction between $\beta$-casein and transglutaminase 2 was disrupted by substitution of the hydrophobic residue to polar residue (Figure IC).

The crystal structure of the transglutaminase 2 : inhibitor complex shows the hydrogen bonding between the inhibitor and the backbone 'NH' of $\mathrm{N} 333 .{ }^{8}$ The carbonyl oxygen of the side chain of the N333 also can interact with the inhibitor. Also, the side chain of Q276 forms a hydrogen bond with the backbone 'NH' of the inhibitor. These two residues are near the acyl-acceptor tumnel of transglutaminase 2 . So. these interactions allow the glutanine residue of the substrate to access the tumnel. In the binding model, the backbone ' $\mathrm{NH}$ ' of the substrate $\mathrm{Q}+1$ position residue interacts with the side chain of $\mathrm{N} 333$, and the interaction is disrupted by substitution of serine (Figure 1D).

Transglutaminase 2 activates NF- $\kappa B$ through polymerization of $\mathrm{I}-\mathrm{kB} \alpha$ and NF- $\mathrm{BB}$ was down-regulated when transglutaminase 2 was inhibited. ${ }^{2.3}$ Therefore. the loss of function by the nutations may be related to the NF- $k B$ down-regulation. We derived models of the transglutaminase 2 complex with three five-amino-acid sequences of I$\mathrm{kB} \alpha$ (Figure 2).

There was no specific interaction between the $\mathrm{Q}+2$ position of the $1 Q^{-66} \mathrm{QQL}$ sequence and the $\mathrm{M} 330$ residue (Figure 2A). But in the I331N mutation. the interaction between the $\mathrm{Q}+3$ position of the substrate was disrupted by substitution to the polar residue, as in the $\beta$-casein model. In the transglutaminase $2: \mathrm{QQ}^{267} \mathrm{QLG}$ and transglutaninase 2: $\mathrm{GQ}^{313} \mathrm{RLT}$ models. there were no specific interactions between the $\mathrm{Q}+3$ position of the substrate and the $133 \mathrm{I}$ residue (Figures 2B, C). However. repulsive interaction between the $\mathrm{Q}+2$ position of the substrate and the arginine residue at the M330 position was found in both models (Figures 2B, C). The disruption of the hydrogen bonding was shown in the N333S mutation for all three $\mathrm{I}-\mathrm{kB} \alpha$ models (Figures 2A, B. C). The interaction between the side chain of N333 and the backbone ' $\mathrm{NH}$ ' of the substrate at the $\mathrm{Q}^{+1}$ position was disnupted by the substitution of serine.

Taking these results together. the residues at the mutation sites strongly contribute to substrate binding in the model.

Depletion of $\mathrm{I}-\mathrm{kB} \alpha$ by transglutaminase 2 in cells. The previous results are based on the in vitro transamidation activity of transglutaminase 2 using the $\beta$-casein substrate. We also tested the polymerization function of transglutaminase 2 in cells in order to compare the in vitro and in wivo conditions. Transglutaminase 2 induces NF- $\mathrm{KB}$ activation through the polymerization of $\mathrm{I}-\mathrm{KB} \alpha^{2}$. And the depletion of free $\mathrm{I}-\kappa \mathrm{B} \alpha$ implies the activation of NF- $\kappa \mathrm{B}$. To test whether transglutaminase 2 mutants lack the $\mathrm{I}-\mathrm{k} \mathrm{B} \alpha$ polymerization function. we analyzed the $\mathrm{I}-\mathrm{kB} \alpha$ level in the cytosolic fraction of transglutaminase 2-transfected PANC-1 cell lines. When the wild-type transglutaminase 2 was expressed in PANC-1 cells the free I- $\kappa \mathrm{B} \alpha$ level was reduced, whereas the M330R. $133 \mathrm{IN}$, and N333S mutants could not deplete the I- $\kappa \mathrm{B} \alpha$ like positive-control C277S mutant (Figure 2). These results are consistent with a the previous report on the
A
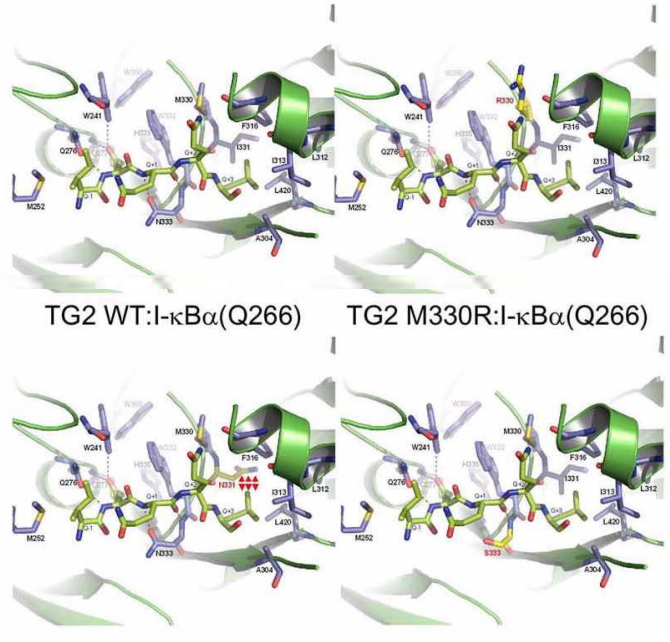

TG2 |331 N:|-kB $\alpha(Q 266) \quad$ TG2 N333S:I-kBa(Q266)

B
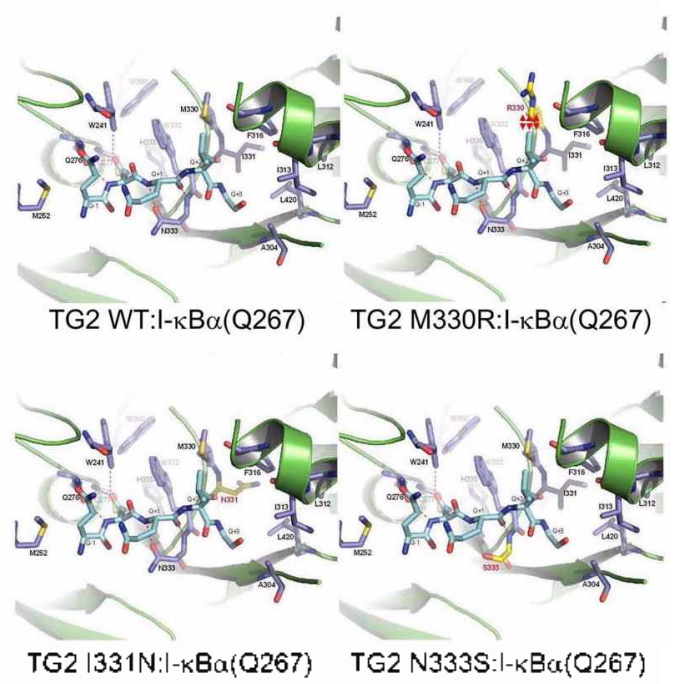

C
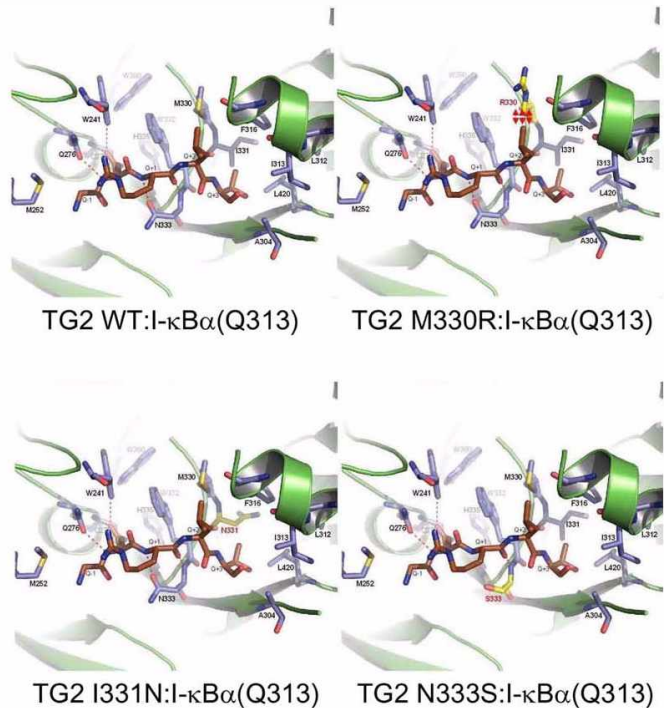

Figure 2. I- $\mathrm{B} \mathrm{B} \alpha$ binding model for three types of mutants. (A) Models for $\mathrm{IQ}^{2 \pi i} \mathrm{QQL}$ sequence of $\mathrm{I}-\mathrm{\kappa B} \alpha$. (B) Models for $\mathrm{QQ}^{20} \mathrm{QLG}$ sequence of $\mathrm{I}-k \mathrm{~B} \alpha$, (C) Models for $\mathrm{GQ}^{3]}{ }^{3} \mathrm{RLT}$ sequence of $I-k B \alpha$. 


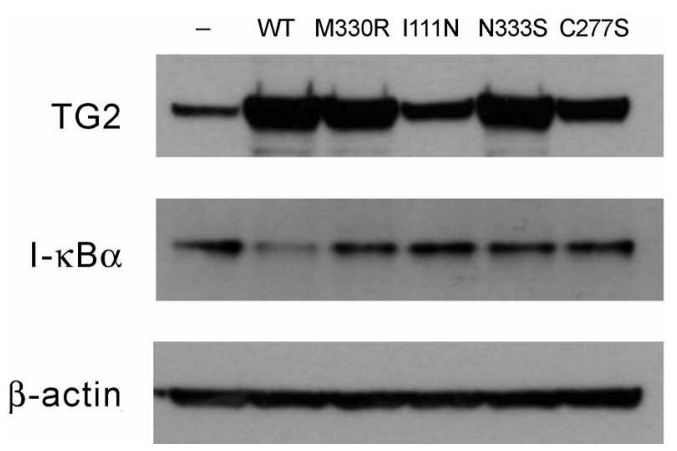

Figure 3. Westem blot analysis of $I-A B \alpha$ and transsglutanninase 2 mutants in PANC-1 cells. The decrease of the I- $\mathrm{AB} \alpha$ level in cry tosol implies the polymerization of $\mathrm{I}-k \mathrm{~B} \alpha$ by transglutaminase 2 . Lane 1: pcDNA 3.0 as mock: lane 2: wild-type transglutanninase 2: lane 3: M330R mutant; lane 4: I33IN mutant: lane 5: N333S mutant; lane 6 : C277S mutant as a positive control. The free I- $\mathrm{kB} \alpha$ level was decreased when the wild type was expressed, whereas the three mutants found in early-onset type 2 diabetes conld not polymerize $\mathrm{I}-\mathrm{AB} \alpha$

transanidation activity of mutants in vitro. ${ }^{6}$ This means that all three transglutaminase 2 nutants in early-onset type 2 diabetes lose their ability for polymerization of I- $\kappa \mathrm{B} \alpha$ which results in NF- $\kappa$ B down-regulation in cells.

In conclusion, transglutanimase 2 mutations in early -onset type 2 diabetes lack protein polymerization activity. The loss of activity by theses mutants was mainly due to the disruption of interaction with the substrate. These nutants do not have the $\mathrm{I}-\mathrm{\kappa B} \alpha$ polymerization function in wo that they have in vitro. The mutants' loss of function can result in down-regulation of NF- $\kappa$ B in pancreatic cells. NF- $\kappa$ B downregulated pancreatic cells night be susceptible to apoptosis. which can cause early-onset type 2 diabetes.

\section{Experimental Section}

Molecular modeling. The reactive glutanine residue of $\beta$-casein was identified from the Transdab data base server (http //genomics.dote.hu/wiki/index.php/Main_Page) ${ }^{7}$ The $\mathrm{IQ}^{260} \mathrm{QQL}, \mathrm{QQ}^{267} \mathrm{QLG}$ and $\mathrm{GQ}^{313} \mathrm{RLT}$ sequences of $\mathrm{I}-\mathrm{KB} \alpha$ were also used for modeling. The conformations of the mutated residues were generated by Rotamer module in Insightll package and the confommation of substrate sequence was optimized with Discover 2000 using the CVFF force field (Accelrys. Inc.). Figure 1 and Figure 2 were made using Py Mol program (http //pymolsourceforge.net). The docking models were determined using the inhibitor (Ac$\mathrm{P}(\mathrm{DON}) \mathrm{LPF}-\mathrm{NH} 2$ ) of X-ray crystal (2Q3Z) structure as a template. ${ }^{8}$ In the substrate sequence $\left(\mathrm{SQ}^{1 \mathrm{~S} 2} \mathrm{SKV}\right)$ of $\beta$-casein. the lysine residue at the $\mathrm{Q}+2$ position was $N, N$ dimethylated to explain the in vitro assay. ${ }^{6}$

Cloning, cell culture and transient transfection of trans- glutaminase 2. Wild-type and mutant human transglutaminase 2 genes were subcloned into pcDNA 3.0 vector (Invitrogen) using the HindIII/Xhol restriction sites. The luman pancreatic cancer PANC-I cell line was grown in DMEM (Hyclone) supplemented with $10 \%$ fetal bovine serum (Hyclone) and 100 uits/mL penicillin-streptonycin (Gibco), and maintained in a humidified $5 \% \mathrm{CO}_{2}$ environment. Transient transfection of pcDNA3.0 containing the fulllength human transglutaminase 2 wild type and the three DNA mutants was performed using the LipofectAMINE 2000 (Invitrogen), according to the manufacturer's instructions. Briefly, the cells were seeded at a density of $2 \times 10^{5}$ per well in a six-well plate and incubated ovemight. The media was replaced by $1 \mathrm{~mL}$ Opti-MEM (Invitrogen) supplemented with DNA-LipofectAMNNE mixture. After 6 hours of incubation, the transfection medium was replaced with fresh culture medium. and the cells were further incubated for 48 hours in a humidified $5 \% \mathrm{CO}_{2}$ environment.

Depletion of $\mathrm{I}-\mathrm{KB} \alpha$ by transglutaminase 2 in cells. We monitored the I- $x \mathrm{~B} \alpha$ level in the cytosol fraction by Western blotting to test the function of the transglutaminase 2 in the cells. ${ }^{2}$ The cytosolic fraction of the samples were prepared using a CelLytic NuCLEAR Extract kit (Sigma). And anti-I$\kappa \mathrm{B} \alpha$ (Cell Signaling), anti-transglutaminase 2 (clone CUB 7402. NeoMarkers) and anti- $\beta$-actin (Abcam) antibodies were used for Western blotting.

Acknowledgments. This work was supported by a National Cancer Center in Korea research grant (grant no. 0710630 and 0810183 )

\section{References}

1. Folk, J. E.; Chung. S. I. Hothods Enzmol 1985. 113,358

2. Lee. J.: Kim, Y. S.: Choi. D. H.: Bang. M. S.: Han. T. R.; Joh, T. H. Kim. S. Y. J. Biol Chem 2004. 279, 53725.

3. Kim. D. S.: Hark. S. S.: Nam. B. H.: Kim. I. H.: Kim. S. Y Cancer Res. 2006. 66. 10936.

4. Kiml. S. Y. Hromt. Biasci. 2006. H. 3026.

5. Kotsakis, P. Griffin, M. Amino Acids 2006, 33. 373.

6. Porzio, O; Massa, O; Cunsolo. V.; Colombo, C: Malaponti, M: Bertuzzi, F.: Hansen, T.: Johansen, A.; Pedersen. O.; Meschi. F.: Teminoni. A.: Melino. G.: Federici. M.: Decarlo. N.: Menicagli. M.: Campani. D.: Marchetti. P.: Ferdaoussi. M.: Froguel. P: Federici. G.: Vaxillaire. M.: Barbetti. F. Hmt. Mutat. 2007.28. 1150.

7. Keresztessy, Z.: Csosz. E: Harstalvi, J.: Csomos, K.: Gray J: Lightowlers, R. N.; Lakey: J. H.: Balajthy. Z.: Fesus. L. Protem Sci. 2006. 15. 2466 .

8. Pitkias. D. M.: Strop. P.: Brunger. A. T.: Khosla. C. PLoS Biol. 2007. 5. 2788 .

9. Park. S. S.: Kim, J. M.: Kim, D. S.: Kim, I. H.: Kim, S. Y. J. Biol. Chem. 2006, 281,34965.

10. Sugimura, Y.: Hosono, M: Wada, F; Yoshimura. T; Maki. M.: Hitomi. K. J. Biol. Chem. 2006. 281. 17699. 